\title{
Maple Syrup Urine Disease and Domino Liver Transplantation: When and How?
}

\section{SEE ARTICLE ON PAGE 889}

Liver transplantation (LT) for various metabolic diseases, for which most patients have a structurally normal liver, has increased the interest in using explanted organs for domino LT. Domino transplantation was initially explored in patients with familial amyloidotic polyneuropathy (FAP), and it is an effective strategy to increase availability of liver grafts and reduce wait-list mortality and dropout, in particular, for LT candidates with low priority. ${ }^{(1)}$

In FAP, as in other rare metabolic diseases, such as primary hyperoxaluria, acute porphyria, and familial hypercholesterolemia, domino LT did not show convincing results because the graft recipients had a risk of developing early or late symptoms related to the underlying enzymatic defect. ${ }^{(1)}$ In contrast, a liver from a patient with maple syrup urine disease (MSUD), a disorder in which the liver does not properly metabolize branched-chain amino acids, can be safely transplanted in a non-MSUD individual because the missing enzyme branched-chain alpha-keto acid dehydrogenase complex is sufficiently expressed in other tissues. ${ }^{(2)}$ Hence, the recipient of

Abbreviations: FAP, familial amyloidotic polyneuropathy; LT, liver transplantation; MSUD, maple syrup urine disease.

Address reprint requests to Marco Spada, M.D., Ph.D., F.E.B.S., Division of Abdominal Transplantation and Hepatobiliopancreatic Surgery, Bambino Gesù Children's Research Hospital, Istituto di Ricovero e Cura a Carattere Scientifico, Piazza Sant'Onofrio 4, 00165 Rome, Italy. Telephone: 003906 68591; FAX: 003906 68591; E-mail:marco.spada@opbg.net

Received April 25, 2019; accepted April 25, 2019.

Copyright (C) 2019 by the American Association for the Study of Liver Diseases.

View this article online at wileyonlinelibrary.com.

DOI 10.1002/lt.25481

Potential conflict of interest: Nothing to report. the domino liver from a patient with MSUD does not have the risk of disease transmission and can tolerate a normal dietary protein intake after LT. However, longterm outcome results still need to be evaluated further. ${ }^{(3)}$

In domino donation, modifications of the surgical technique (greater care for the liver explant, possible longer time of surgery and anesthesia, the level of vessel transection, and any need of hepatic venoplasty) and a modification of the preoperative management that is required to obtain a transplantable liver graft (some programs use anticoagulation in the MSUD domino donor $^{(3)}$ ) should not expose the MSUD patient to additional risks. Although there are not yet data comparing LT outcomes in MSUD domino donors versus nondonors, the European data showed no differences in morbidity and survival rates in the FAP patients. ${ }^{(4)}$

In this issue of Liver Transplantation, Herden et al. ${ }^{(5)}$ describe the outcomes of 15 LTs in MSUD patients (12 children and 3 adults) from whom 14 grafts were used for domino LT in the Eurotransplant area. After 23 months of follow-up, the patient survival rates were 100\% for MSUD recipients (all on a free diet) and 93\% for domino LT recipients (1 MSUD liver recipient died from tumor recurrence). Surgical complication rates were similar to those reported for standard transplantation. These excellent results support recent data from a propensity-matched analysis of the United Network for Organ Sharing registry ${ }^{(1)}$ showing that domino LTs $(n=123)$ have similar 10-year graft and patient survival rates compared with deceased donor LT. Despite the technically challenging implantation procedure, ${ }^{(5)} 2$ domino liver grafts of the Eurotransplant series underwent an ex situ split procedure, and the derived 4 partial grafts were successfully transplanted, reflecting excellent MSUD graft quality. Moreover, the good outcomes of domino liver grafts shipped to other centers of the Eurotransplant area support that intercenter domino organ sharing does not affect domino LT outcomes despite prolonging ischemia times.

In the Eurotransplant network, ${ }^{(5)}$ the presence of an intercenter allocation system facilitated allocation of 
$80 \%$ of domino liver grafts after a median of 5 (range, 3-18) refusals before acceptance by standard allocation rules in 53\% of the patients. Three (20\%) MSUD organs were discarded for domino LT because of the lack of size-matching recipients (donor age, 4 and 6 months and 2 years), whereas none were discarded for technical issues.

Because LT has been proven to be the elective treatment for severe MSUD patients by providing increased patient survivals and quality of life, ${ }^{(6)}$ the timing of LT should not wait for episodes of metabolic decompensation and/or the occurrence of neurological damage, but it should be undertaken before the development of MSUD complications. Consequently, domino liver grafts from MSUD patients will derive more and more frequently from very small patients. As for MSUD LTs, small-sized domino liver grafts will come also from children with other metabolic diseases, for which early LT is recommended. ${ }^{(7)}$

As documented by Herden et al., (5) half of MSUD domino liver grafts did not follow the regular allocation system but were allocated on the basis of size matching, preferentially choosing patients belonging to disadvantaged weight (neonates or adolescents) and low Pediatric End-Stage Liver Disease score. Hence, with domino LT, the transplant community will have to review and update allocation rules for domino liver grafts in order to maximize the use of this resource. When a MSUD patient is listed for LT, it is possible to estimate liver volumetry and anatomical data, and therefore, a potential domino recipient should be identified at a national or international level. Despite lingering concerns about the use of living donor LT in MSUD patients, ${ }^{(5,8)}$ it now offers additional options for domino LT allocation.

\author{
Marco Spada, M.D., Ph.D., F.E.B.S. (D) 1 \\ Roberta Angelico, M.D., Ph.D., F.E.B.S. (D) 1 \\ Carlo Dionisi-Vici, M.D. ${ }^{2}$ \\ ${ }^{1}$ Division of Abdominal Transplantation and \\ Hepatobiliopancreatic Surgery \\ ${ }^{2}$ Division of Metabolism \\ Bambino Gesù Children's Hospital IRCCS \\ Rome, Italy
}

\section{REFERENCES}

1) Geyer ED, Burrier C, Tumin D, Hayes D Jr, Black SM, Washburn WK, Tobias JD. Outcomes of domino liver transplantation compared to deceased donor liver transplantation: a propensitymatching approach. Transpl Int 2018;31:1200-1206.

2) Khanna A, Hart M, Nyhan WL, Hassanein T, Panyard-Davis J, Barshop BA. Domino liver transplantation in maple syrup urine disease. Liver Transpl 2006;12:876-882.

3) Schenck D, Mazariegos GV, Thistlethwaite JR Jr, Ross LF. Ethical analysis and policy recommendations regarding domino liver transplantation. Transplantation 2018;102:803-808.

4) Tincani G, Hoti E, Andreani P, Ricca L, Pittau G, Vitale V, et al. Operative risks of domino liver transplantation for the familial amyloid polyneuropathy liver donor and recipient: a double analysis. Am J Transplant 2011;11:759-766.

5) Herden U, Grabhorn E, Santer R, Li J, Nadalin S, Rogiers X, et al. Surgical aspects of liver transplantation and domino liver transplantation in maple syrup urine disease: analysis of 15 donor-recipient pairs. Liver Transpl 2019;25:889-900.

6) Mazariegos GV, Morton DH, Sindhi R, Soltys K, Nayyar N, Bond $\mathrm{G}$, et al. Liver transplantation for classical maple syrup urine disease: long-term follow-up in 37 patients and comparative United Network for Organ Sharing experience. J Pediatr 2012;160:116-121.

7) Fagiuoli S, Daina E, D’Antiga L, Colledan M, Remuzzi G. Monogenic diseases that can be cured by liver transplantation. J Hepatol 2013;59:595-612.

8) Roda KMO, Vincenzi R, Fonseca EA, Benavides M, Turine P, Afonso RC, et al. Domino liver transplant in maple syrup urine disease: technical details of cases in which the first surgery involved a living donor. Transplantation 2019;103:536-543. 